(Aus dem physiologischen Institut der Universität Marburg.)

\title{
Zur Theorie der Farbenempfindung.
}

Von

\section{F. Schenck.}

Die soeben erschienene Abhandlung Bernstein's'): "Eine neue Theorie der Farbenempfindung" zeigt in einigen Punkten Anklänge an eine Auffassung, die ich mir in letzter Zeit gebildet, habe, und die ich demnächst in einer ausführlichen Publikation zu begründen suchen wollte. Mit Rücksicht auf das Interesse, das die Frage durch die Publikation Bernstein's hervorruft, will ich meine Auffassung auch schon kurz bekannt geben.

Ich habe bisher zu der Fick'schen Theorie der Farbenblindheit geneigt, weil diese unzweifelhaft gewisse Vorteile gegenüber anderen Theorien bietet. Aber die Fick'sche Theorie bedarf gewisser Einschränkungen und Ergänzungen, um allen Tatsachen gerecht zu werden, insbesondere den Tatsachen, dass die Helligkeitsverteilung im Spektrum bei manchen Formen von Farbenblindheit anders ist wie beim Trichromaten und Deuteranopen, und dass die Neutralpunkte bei den beiden Formen von Rot-Grün-Blindheit nicht weit voneinander entfernt liegen. Auch muss der Grundgedanke der Fick'schen Theorie so umgestaltet werden, dass die Theorie entwicklungsgeschichtlich begreiflich wird.

Ich nehme an, dass der Zapfenapparat in einem frühen Entwicklungsstadium nur eiıe Sebsubstanz enthält, deren Erregung die Empfindung Weiss vermittelt, und die auch insofern der Stäbchensehsubstanz nahe steht, als sie gegen langwellige Lichter verhältnismässig wenig empfindlich ist.

Die Zapfensubstanz erleidet nun zunächst eine Veränderung, die ich in Anlehnung an die photographische Nomenklatur Panchromatisation nennen will, und die darin besteht, dass die Substanz empfindlicher

1) Naturwiss. Rundschau Bd. 21 Nr. 38. 
für langwellige Lichter wird. Dieser Vorgang besteht vielleicht in der Entwicklung eines Sensibilisators für langwellige Lichter.

Die weiteren Entwicklungsvorgänge bestehen in Teilungen der ursprünglichen Sehsubstanz, verbunden mit Differenzierung der entstehenden Teile, und zwar:

1. Zunächst entstehen aus der ursprünglicben die Weissempfindung vermittelnden Sehsubstanz durch Teilung zwei Substanzen, deren eine vorwiegend durch langwellige Lichter erregbar die Empfindung Gelb, deren andere vorwiegend dureh kurzwellige Lichter erregbar die Empfindung Blau vermittelt; dabei haftet den beiden Teilen $\mathrm{zu}$ sammen aber insofern noch die Eigenschaft ihrer Muttersubstanz an, als ihre gleichzeitige Errégung "in der Stärke, wie sìe durch das gemischte Tageslicht erfolgt, "die Weissempfindung hervorruft:

2. "In analoger Weise teilt sich danach die Substanz, welche die Gelbempfindung vermittelt, in eine die Rotempfindung und eine die Grünempfindung hervorrufende Substaniz, deren"gleichzeitige Erregung wieder die Empfindung ihrer Muttersubstanz, d. i. Gelb, bewirkt.

Für die Erklärung der angeborenen Farbenbliridheit ist noch anzunehmen, dass die Teilungsvorgänge unabhängig von der Panchroma tisation, also auch ohne Vorausgehen der letzteren, statthaben könnens

Dann sind folgende Arten von Farbenblindheit theoretisch zú konstruièren:

I. Totale Farbenblindheit; sie besteht:

1. entweder auf völlständigem Fehlen der Zapfenfunktion: reines Stäbchensehen, charakterisiert durch die dem Dämmerungsssehen entsprechende Helligkeitsverteilung im Spektrum und zentrales Skotom;

2. oder auf einem Ausbleiben der Teilungsvorgänge; hier sind noch zwei Fälle zu unterscheiden:

a) ohne Panchromatisation; dahin 'gehören, vielleicht die von Hess und v. Hippel beschriebenen Fälle totaler Färbenblindheit ohne zentrales Skotom; ferner gehört hierher die totale Farbenblindheit in der äussersten Netzhautzone des Protanopen;

b) mit Panchromatisation; dahin gehört die totale Farbenblind-: heit der äussersten Netzhautzone des Trichromaten und des Deuteranopen; sie ist charakterisiert durch die dem Hellsehen des Triehro-; maten entsprechende Helligkeitsverteilung im Spektrum; ,0b sie aueh $_{3}$ jemals auf der ganzen Netzhaut gefunden worden ist, vermag ich den Literaturangaben nicht $\mathrm{zu}$ entnekmen. 
II. Rot-Grün-Blindheit; sie beruht auf dem Ausbleiben der Teilung der "Grelbsubstanz" in die "Rotsubstanz" und die "Grünsubstanz". Hier sind zwei Fälle möglich:

1. ohne Panchromatisation: Protanopie; die "Gelbsubstanz" ist in diesem Falle, wie ihre Muttersubstanz, unempfindlich für rotes Licht;

2. mit Panchromatisation : Deuteranopie und die Rot-Grün-Blindheit der mittleren Netzhautzone des Trichromaten.

Jeder der Vorgänge der Panchromatisation und der Teilungen läuft, wenn er einmal begonnen hat, in der Regel auch bis zu Ende ab. Daraus erklärt sich, dass die Farbenblindheit in bestimmten typischen Formen auftritt, zwischen denen keine Übergänge existieren.

Nur ausnahmsweise kann es vorkommen, dass die Teilung eine unvollkommene ist, so dass Verbindungen zwischen den Teilen übrig bleiben, welche die Erregung in abgeschwächtem Masse von einem Teil auf den anderen übertragen: Daraus erklärt sich z. B. die selten vorkommende Blau-Gelb-Blindheit, bei der infolge einer mangelhaften Teilung die "Rotsubstanz" und die "Grünsubstanz" noch in Verbindung sein mögen mit der "Blausubstanz", und zwar so, dass infolge einer gewissen Irreziprozität der Erregungsleitung in den Verbindungen die Erregung von der "Blausubstanz" leichter auf die "Rotsubstanz" und die "Grünsubstanz" übergeleitet werden kann als von der "Rotsubstanz" auf die "Blausubstanz" und danach auf die "Grünsubstanz", und als von der "Grünsubstanz" auf die "Blausubstanz" und danach auf die "Rotsubstanz".

Dis anomale Trichromasie dagegen scheint mir mehr durch mangelhafte Ausbildung des einen oder anderen Teiles als durch mangelhafte Teilung bedingt zu sein.

Unsere Theorie nimmt wie die Young-Helmholtz'sche für das ausgebildete Auge Trichromasie an. Gegenüber der Lehre, welche die Dichromasie durch Fehlen einer der drei Grundempfindungen zu erklären sucht, bietet aber meine Theorie den Vorteil, dass sie zwei Tatsachen leichter erklärt: nämlich erstens die Tatsache, dass bei der sogenannten Rot-Blindheit nicht die Grundempfindungen Grün und Blau und bei der sogenannten GrünBlindheit nicht Rot und Blau; sondern in beiden Fällen Gelb und Blau übrig bleiben, und zweitens die Tatsache, dass die Helligkeitsverteilung im Spektrum für den Deuteranopen mit der für den Trichromaten übereinstimmt; nicht aber für den Protanopen. 
Der Hering'schen Theorie habe ich denjenigen Gedanken entlehnt, der gerade die Stärke dieser Theorie ausmacht, d. i. die Auffassung, dass - wenn auch nicht wie Hering meint, im fertig entwickelten Auge, so doch auf einer frühesten Entwicklungsstufe auch im Zapfenapparat eine die Weissempfindung (resp. in Ruhe Schwarzempfindung) vermittelnde Sehsubstanz vorbanden ist, dass ferner - wenigstens auf einer mittleren Entwicklungsstufe - der Zapfenapparat die Empfindungen Gelb und Blau (ohne Rot und Grün) vermittelt, und dass die Empfindungen Gelb und Blau sich gegensätzlich verhalten, weil sie zusammen Weiss liefern. Gegenüber der Hering'schen Theorie wird meine Theorie aber besser der Tatsache gerecht, dass es zwei Arten von Rot-Grün-Blindheit gibt. Auch fallen bei meiner Theorie die Schwierigkeiten weg, die in der Annahme einer Assimilationserregung liegen.

Auch das Prinzip der Fick'schen Theorie ist, wenn auch etwas umgestaltet, in meiner Theorie wiederzuerkennen, soweit es sich um die Erklärung der Deuteranopie und der totalen Farbenblindheit der äussersten Netzhautzone handelt. Fick nimmt für die Deuteranopie eine Übereinstimmung der Erregbarkeit der Rot- und der Grünsubstanz an, für die genannte Form der totalen Farbenblindheit dagegen eine Übereinstimmung der Erregbarkeit aller drei Sehsubstanzen; ich führe diese Übereinstimmung darauf zurück, dass bei jenen Formen der Farbenblindheit die zwei resp. drei Substanzen noch in ibrer Muttersubstanz vereinigt sind und daher nicht unabhängig voneinander, sondern zusammen, also immer gleichzeitig und gleichstark erregt werden. Betreffs der Erklärung der anderen Formen von Farbenblindheit weiche ich von Fick ab, aber in diesem Punkte war die Fick'sche Theorie auch nicht binreichend.

Mit Bernstein habe ich gemeinsam, dass wir beide bestrebt sind, die Farbenempfindung und Farbenblindheit entwicklungsphysiologisch unserem Verständnis näher zu bringen; ich habe aber gegenüber Bernstein den Vorteil, dass ich mit einer geringeren Zahl von Sehsubstanzen und auch sonst in einfacherer Weise zurecht komme.

Meine Theorie wird auch der auf Grund psychologischer Überlegungen öfter ausgesprochenen Ansicht gerecht, dass jeder sogenannten einfachen oder reinen Empfindung ein besonderer physiologischer Prozess zugrunde liegt. Für Rot, Grün und Blau gilt das von dem fertig entwickelten Auge, für Gelb und Weiss von früheren 
Entwicklungsstufen. Wenn Gelb später auf zwei, Weiss auf drei Grundprozessen beruht, so liegt das nur daran, dass die zwei resp. drei Prozesse durch Entwicklung sich aus ursprünglich einfachen Prozessen ausgestaltet haben. Dem psychologischen Postulate wird aber meine Theorie insofern auch gerecht, als sie für das wirklich reine Rot und das reine Grün je einen besonderen Grundprozess annimmt, während Hering auf Grund dessen, was die Untersuchung der partiell farbenblinden Netzhautzone ergeben hat, den besonderen Vorgang für ein bläuliches Rot und ein bläuliches Grün annehmen müsste.

Ich kenne keine Tatsache, die meiner Theorie widerspricht. Dagegen lassen sich gerade die wichtigsten Sätze der Lebre von der Farbenblindheit leicht und ungezwungen aus dieser Theorie erklären. 